Manuelle Medizin 2003 · 41:301 DOl 10.1007/s00337-003-0232-1

Online publiziert: 28 Juni 2003

(c) Springer-Verlag 2003

H. Moll · Leutkirch

\title{
Manuelle Medizin in der Sportmedizin
}

turelle Zuordnung der Störung zu. Die segmentale Bewegungsprüfung mit Reaktion des nozireaktiven muskulären $\mathrm{Hy}$ pertonus auf die Bewegung weist uns bei funktionellen Störungen (Blockierungen) den Weg zur Therapie.

Zum Ausschluss struktureller Veränderungen wie Traumafolgen, Anomalien oder möglichen Instabilitäten sind Röntgenaufnahmen, in der Regel in 2 Ebenen, obligate Voraussetzung vor einem chirotherapeutischen Eingriff. Weiterführende apparative bildgebende Verfahren bzw. Labordiagnostik schließen sich in Abhängigkeit von Vorbefunden an.

Prädisponiert für funktionelle Störungen an der Wirbelsäule sind Sportarten mit rascher Beschleunigung bzw. Verzögerung oder Stauchung einzelner Wirbelsäulenabschnitte wie Wurfdisziplinen, Tennis, Turnen oder Sprungdisziplinen. Ebenso ungünstig können sich rasche Rotationsbewegungen, wie sie beim Golfspiel, Tennis oder bei Wurfsportarten auftreten, auswirken. Unphysiologische Beanspruchung in der Sagittalebene wie Kunstturnen, Turmspringen oder Delphinschwimmen zeigen sich anfällig für Blockierungen, länger dauernde repetitive Belastungen münden bei mangelnder Therapie nicht selten in strukturellen Veränderungen.

Häufige Verletzungen der Gliedmaßen betreffen vor allem Schulter-, Knie- und Sprunggelenk. Die manualmedizinische Diagnostik lässt in vielen Fällen gemeinsam mit Anamnese und Inspektion eine zuverlässige Aussage über das Ausmaß der Verletzung zu. Auf bildgebende Verfahren (Röntgen, MRT, Sonographie) kann aber in der Regel - nicht zuletzt auch we- gen der Dokumentationspflicht - nicht verzichtet werden.

„Blockierungen“ ohne strukturelle Läsion wie z. B. des Fibulaköpfchens oder des oberen/unteren Sprunggelenks sind ebenso Gegenstand manueller Therapie wie der Einsatz solcher Techniken in der Nachbehandlung konservativ oder operativ versorgter Verletzungsfolgen.

Die Kenntnis von Verkettungssyndromen, beispielhaft seien hier der Zusammenhang des peripatellären Schmerzsyndromes bzw. der Epikodylopathie mit Störungen der LWS bzw. unteren HWS/mittleren BWS genannt, sind Voraussetzung für eine erfolgreiche Behandlung solcher Erkrankungsformen, die sich nicht selten in einem eher diffusen, nicht klar abgrenzbaren Beschwerdebild äußern.

Die Behandlung sportbedingter Verletzungen und Überlastungsschäden umfasst neben der Manualmedizin mit manipulativen und mobilisierenden Techniken auch infiltrative sowie krankengymnastisch-physikalische Maßnahmen. Trainingssteuerung mit Beratung des Sportlers hinsichtlich Sportgerät und Kleidung setzt entsprechende Kenntnisse voraus, ebenso die Versorgung mit orthopädietechnischen Hilfsmitteln und Schuhzurichtungen für Korrekturen der Statik.

\section{Korrespondierender Autor}

\section{Dr. H. Moll}

Blaichstr. 10, 88299 Leutkirch E-Mail: DrHorstMoll@aol.com prüfung an. Pathologien an der Wirbelsäule lassen mittels topischer Palpation, segmentaler Bewegungsspielprüfung und Testung des (nozireaktiven) Muskeltonus nicht selten bereits eine lokale und struk- 